
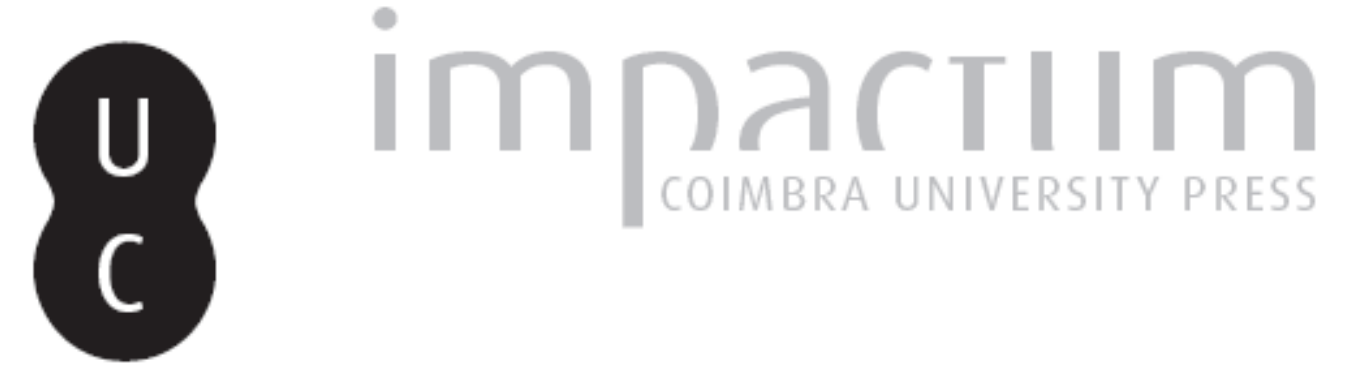

\title{
[Recensão a] Note critique: One Book. The Whole Universe: Plato's Timaeus today, Richard D. Mohr, Barbara M. Sattler (ed.)
}

\author{
Autor(es): Brisson, Luc
}

Publicado por: Imprensa da Universidade de Coimbra

URL persistente:

URI:http://hdl.handle.net/10316.2/42190

DOI:

DOI:https://doi.org/10.14195/2183-4105_11_11

Accessed : $\quad$ 26-Apr-2023 11:53:46

A navegação consulta e descarregamento dos títulos inseridos nas Bibliotecas Digitais UC Digitalis, UC Pombalina e UC Impactum, pressupõem a aceitação plena e sem reservas dos Termos e Condições de Uso destas Bibliotecas Digitais, disponíveis em https://digitalis.uc.pt/pt-pt/termos.

Conforme exposto nos referidos Termos e Condições de Uso, o descarregamento de títulos de acesso restrito requer uma licença válida de autorização devendo o utilizador aceder ao(s) documento(s) a partir de um endereço de IP da instituição detentora da supramencionada licença.

Ao utilizador é apenas permitido o descarregamento para uso pessoal, pelo que o emprego do(s) título(s) descarregado(s) para outro fim, designadamente comercial, carece de autorização do respetivo autor ou editor da obra.

Na medida em que todas as obras da UC Digitalis se encontram protegidas pelo Código do Direito de Autor e Direitos Conexos e demais legislação aplicável, toda a cópia, parcial ou total, deste documento, nos casos em que é legalmente admitida, deverá conter ou fazer-se acompanhar por este aviso.

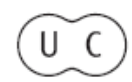




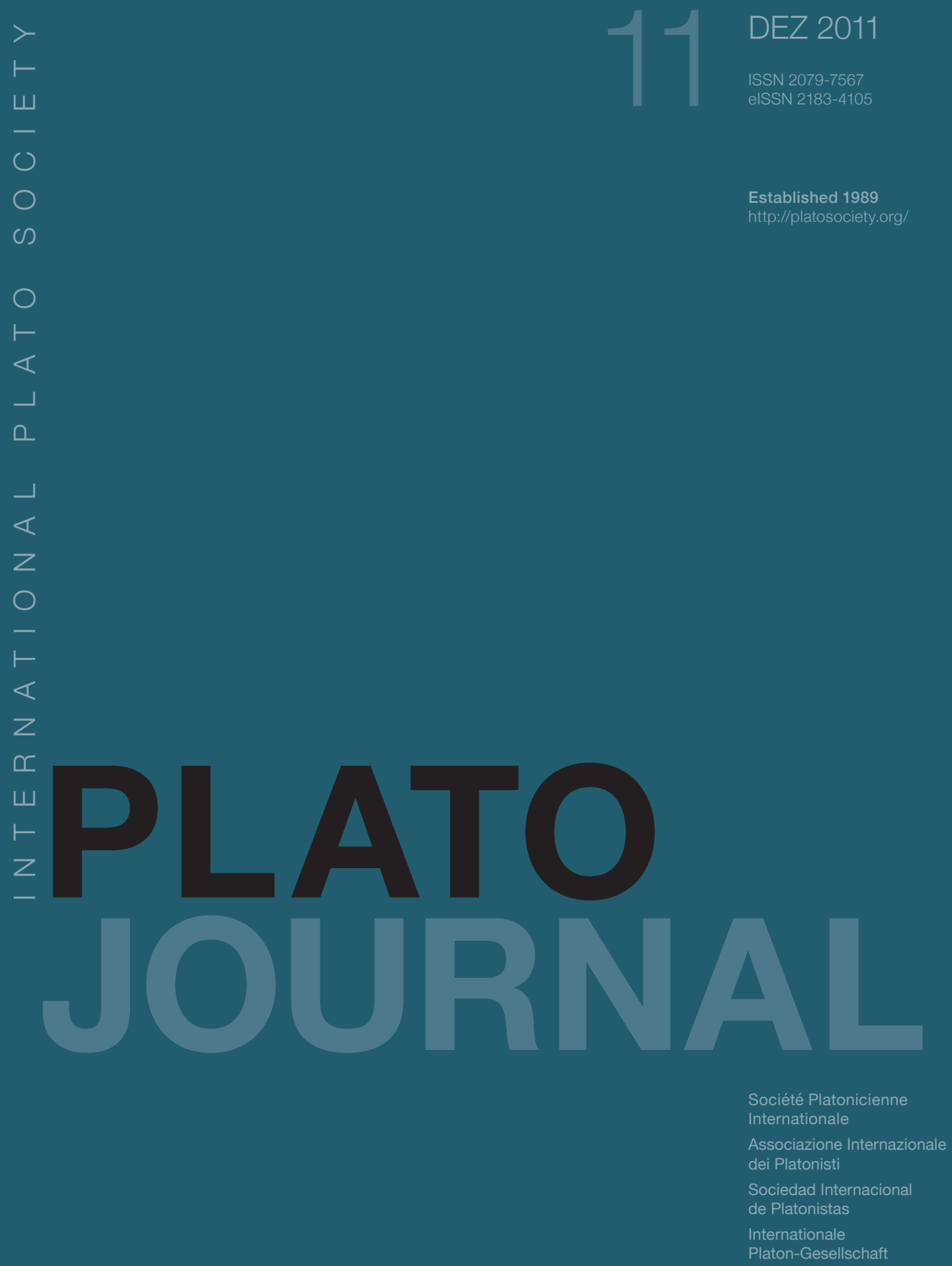




\title{
NOTE CRITIQUE
}

\author{
One Book. The Whole Universe: Plato's Timaeus today, \\ Richard D. Mohr, Barbara M. Sattler (ed.), Las Vegas / \\ Zürich / Athens, Parmenides publishing, 2010 viii-406 p.
}

Le volume consiste en un recueil d'articles sur le Timée, ou plutôt sur la réception du Timée depuis l'Antiquité jusqu'à nos jours, comme l'indique son sous-titre. Y interviennent non seulement des historiens de la philosophie dans l'Antiquité, mais aussi divers spécialistes, dont Anthony Legget, Prix Nobel de Physique 2003 ; le livre bénéficie aussi d'un easy-to-navigate companion website http://www.parmenides.com/Timaeus/pages/supplement.html, où l'on trouve des versions animées de certains diagrammes et d'extraits de films sur l'Atlantide. Tous les auteurs ont participé à une rencontre interdisciplinaire qui s'est tenue à l'Université d'Illinois (Urbana) en 2007, et qui avait pour titre. «Life, the Universe, Everything - and more: Plato's Timaeus today ». La première partie du titre semble avoir été suggérée par le fait que, dans un seul « livre » de 65 pages (éd. Estienne), le Timée donne un exposé concernant «a comprehensive theology, metaphysics, physics, epistemology, and psychology, with significant excursions into logic, biology, astronomy, medecine, and ethics. » (p. 1)

Le livre se compose de sept sections: I. The big question, qui ne comprend que quelques pages par Anthony J. Legget ; II. God and related matter, qui porte sur la démiurgie et la place du Timée dans l'œuvre de Platon; III. Space, place, and motion: The receptacle of becoming qui a pour objet la «matière »; IV. Aristotle's Timaeus; V. Reason and myth, qui comporte deux articles discutant d'un article de M. Burnyeat sur l'eikos muthos; VI. Time, narrative and myth, qui s'intéresse au temps dans la réalité et dans le récit; VII. Timaean architecture: Timber framing the universe and building today, qui établit des rapports entre le Timée et l'architecture moderne; et enfin VIII. Ever after, ever before, qui ne comprend qu'un article, celui de l'astrophysicien Sean M. Carroll: « Time and change in an eternal universe » portant en fait sur le Big Bang.

PLATO, The electronic Journal of the International Plato Society, n 11, 2011.

http://gramata.univ-paris1.fr/Plato/article108.html

(c) All rights of reproduction of any form reserved. 
L'intérêt de ce recueil, on l'a dit en commençant, réside dans le fait qu'il s'intéresse à la réception du Timée, et cela non seulement dans l'Antiquité (Aristote, les Stoïciens, Plutarque, les Médio-platoniciens et les NéoPlatoniciens), mais aussi à l'époque moderne et contemporaine (les tentatives de récupération du mythe de l'Atlantide, Derrida, l'architecture, la physique moderne et l'astrophysique). Tous ces sujets présentent un véritable intérêt. Mais on remarque que, à l'exception des articles de Charles Kahn et de Thomas Robinson sur la place du Timée dans l'œuvre de Platon, et de ceux de Donald Zeyl et de Verity Harte sur la «matière », on trouve peu de choses sur le Timée lui-même. L'article de Stephen Menn porte sur l'arrière plan présocratique du dialogue, l'article d'Allan Silverman, celui de Thomas Johansen et celui d'Alan Code portent sur la lecture du Timée par Aristote ; l'article d'Anthony A. Long sur celle du Timée par les Stoïciens, l'article de Matthias Vorwerk sur celle du Timée par Plutarque, celui de Gabor Betegh et celui d'Alexander P.D. Mourelatos sur une interprétation «médio-platonicienne » du rapport entre démiurge et les Formes dans le Timée par Myles Burnyeat (on verra plus loin en quel sens le qualificatif est utilisé), et celui de Ian Mueller sur une lecture néoplatonicienne du Timée.

Cette absence d'intérêt pour le dialogue en lui-même illustre bien la spécificité de l'approche anglo-américaine de Platon en général et du Timée en particulier, l'un des dialogues les plus tenus à l'écart, en raison de ses aspects mythologiques, théologiques et métaphysiques. Les Formes, la figure du démiurge et le rôle de la khora ne se sont jamais remis de la critique aristotélicienne. Seule une interprétation stoïcienne qui réintègre la divinité fabricatrice dans le monde, et une interprétation médio-platonicienne qui fait des Formes les pensées de la divinité suprême rend le dialogue « acceptable » d'un point de vue philosophique pour les interprètes contemporains. Les Formes, le démiurge et la khora sortent du champ des préoccupations de ceux qui refusent de s'aventurer au-delà d'un monde physique clos sur lui-même. Ce premier enfermement en révèle un autre non seulement dans la langue anglaise, mais aussi et surtout dans un contexte académique ne reflétant que les préoccupations de quelques grandes universités anglaises et américaines. Ce double renfermement ne peut que faire dégénérer les recherches sur l'histoire de la Philosophie dans l'Antiquité

Une telle réserve pourrait s'expliquer par le fait que le Timée constitue une pierre d'achoppement pour l'interprétation dominante depuis l'après-guerre. La présence de la doctrine des Formes, l'intervention d'une divinité comme le

PLATO, The electronic Journal of the International Plato Society, n 11, 2011.

http://gramata.univ-paris1.fr/Plato/article108.html

(c) All rights of reproduction of any form reserved. 
démiurge et celle de l'âme du monde qui en prend le relais constituaient autant d'éléments «non-philosophiques » qu'il convenait d'évacuer. On tenta d'abord de situer la composition du dialogue avant celle du Parménide, dialogue qui marquerait l'abandon par Platon de la théorie des Formes, puis on chercha à montrer que l'on pouvait expliquer le dialogue sans tenir compte des Formes, du démiurge et de l'âme.

Les articles de Charles Kahn et de Thomas Robinson portent les traces de la première tentative. Dans « The place of cosmology in Plato's later dialogues », Charles Kahn maintient l'interprétation unitarienne suivant laquelle les dialogues de vieillesse maintiennent les éléments essentiels de la doctrine des dialogues de la maturité, tout en y apportant certains aménagements tenant compte des critiques d'Aristote. Dans un article au titre énigmatique: «Plato on (just about) everything. Some observations on the Timaeus and other dialogues », Thomas M. Robinson se situe dans le même contexte interprétatif, mais en insistant sur le rapport entre cosmologie et justice qui l'amène du côté de la modernité, en évoquant le Big Bang et l'œuvre de J. Rawls.

On peut vérifier la persistance de la tentative d'interpréter le Timée de Platon sans tenir compte de ces éléments non-philosophiques que sont les Formes, le démiurge et l'âme du monde dans les articles qui portent directement sur le dialogue. Stephen Menn cherche à montrer que, dans le Timée, Platon s'oppose à l'explication d'Empédocle suivant laquelle l'apparition d'entités physiques distinctes s'explique par un processus centrifuge initié par le Noûs; cet essai, brillant, reste très spéculatif, car il se fonde sur une interprétation d'Anaxagore qui reste discutable, et sur l'hypothèse suivant laquelle Platon dans le Timée comme Socrate dans le Phédon s'en prend à Anaxagore. Comme l'indique le titre de son article: «Visualizing Platonic space », Donald Zeyl commence par se représenter la khora, comme un espace rempli de matière, qu'il considère comme un fluide malléable; d'où l'image qui revient souvent de l'aquarium dans lequel se déplacent des poissons. Dès lors, on doit se représenter les éléments dont sont faites les choses sensibles non comme des grains de matière se déplaçant dans un espace vide, mais comme des configurations et des reconfigurations de masses déterminées dans et à partir d'une matière fluide ; je ne puis qu'être d'accord avec cette façon de voir la khora, car, dans mon commentaire systématique sur le Timée publié en $1974\left(1995^{2}, 1998^{3}\right)$, et que j'ai repris dans un article en anglais ("How and why do the building blocks of the universe change constantly in Plato's

PLATO, The electronic Journal of the International Plato Society, n 11, 2011.

http://gramata.univ-paris1.fr/Plato/article108.html

(C) All rights of reproduction of any form reserved. 
Timaeus (51a-61c) ?", Plato Physicus. Cosmologia e antropologia nel Timeo, a cura di Carlo Natali e Stefano Maso, Amsterdam (Hakkert) 2003, p. 189-204), j'avais montré que la khora n'était pas seulement ce dans quoi se trouvaient les éléments mais aussi ce de quoi ils étaient faits, d'où la formule que je proposais pour la nommer: «milieu spatial ». L'intérêt de l'article de D. Zeyl est d'illustrer ce type d'interprétation qui ne réduit pas la khora à un espace vide. Verity Harte aborde le même thème dans un article intitulé: « The receptacle and the primary bodies. Something from nothing? », mais en s'attachant à une autre façon de voir la khora, comme une tablette de cire sur laquelle sont imprimés les éléments. Dès lors, pour elle, les polyèdres réguliers ne sont ni des configurations dans l'espace (considéré comme un récipient), ni des configurations faites d'espace (comme une statue est faite de bronze), mais des configurations de l'espace. Tout le problème est de savoir en quel sens il faut prendre l'expression « configuration of space », l'espace étant ainsi défini : «Space, abstractly conceived, is the (unstructured) medium informed by the specific geometrical configuration of each regular solid. » (p. 136). Cela dit, Verity Harte fait une remarque très intéressante qui explique le deuxième membre de son titre: étant donné que la khora comporte des traces des éléments avant l'intervention (temporelle ou fictive) du démiurge, on ne peut parler de création à partir de rien. Pour sa part, Zina Giannopoulou s'attaque à l'interprétation de la khora proposée par Derrida: «Derrida's khôra, or unnaming the Timaean receptable», suivant laquelle la nécessité d'introduire dans le Timée la khora, décrite à l'aide d'un mélange confus de métaphores, prouve que le dualisme Formes / choses sensibles est intenable. Zina Giannopoulou montre que l'introduction de la khora permet de résoudre le problème du dualisme, et surtout que les métaphores utilisées pour décrire cette étrange entité sont tout à fait appropriées ; il convient de noter la richesse de la littérature secondaire à laquelle fait appel l'auteur. Allan Silverman s'intéresse pour sa part au démiurge dans « Philosopher-kings and craftsman-gods », assimilant le démiurge du Timée au dirigeant de la cité dans la République. Il s'interroge sur les rapports entre la raison théorique et la raison pratique qu'Aristote aborde dans l'Éthique à Nicomaque et dans la Métaphysique; le motif qui pousse et le dirigeant politique et le démiurge est la recherche du bien. Il est surprenant qu'Allan Silverman ne semble pas connaitre l'article qu'André Laks a fait paraitre plusieurs fois, et notamment sous ce titre « Legislation and demiurgy : on the relationship between Plato's Republic and Laws », Classical Antiquity 9, 1990, 209-229.

PLATO, The electronic Journal of the International Plato Society, n 11, 2011.

http://gramata.univ-paris1.fr/Plato/article108.html

(c) All rights of reproduction of any form reserved. 
On remarquera qu'on retrouve tout au long de ce recueil des allusions à l'article de Myles Burnyeat: «Eikos mythos», présenté oralement depuis les années 2000, publié pour la première fois dans la revue bulgare Rhizai 2, 2005, p. 143-165, puis réimprimé dans Catalin Partenie (Plato's Myths, Cambridge / New York (Cambridge University Press) 2009); une bonne preuve de l'utilité des réseaux. Dans cet article, Myles Burnyeat veut montrer que le Timée est bien un mythe sur l'origine de l'univers, à la façon d'Hésiode dans la Théogonie, mais que le qualificatif eikos ne doit par être pris dans le sens habituel de « vraisemblable », indiquant que l'on parle des choses sensibles qui ne sont que les images de ces réalités véritables que sont les Formes, mais dans un sens normatif, comme le fait Cicéron, qui parle d'《approprié $»$, de « raisonnable $\gg$. Dans un article à paraître («Why is the Timaeus called an eikôs muthos», Plato and myth, edited by Catherine Collobert, Pierre Destrée and Francisco J. Gonzalez, to be published, Leiden, Brill, 2012), j'exprime mon accord avec la position de Myles Burnyeat sur la question du mythe, mais mon désaccord sur la question de l'eikos. Une analyse et une traduction des 16 passages du Timée où apparaît eikos ne laisse aucun doute : l'opposition choses sensibles / réalités intelligibles est bien présente dans tous les cas. Alexander Mouraletos, « The epistemological section (29b-d) of the Proem in Timaeus' speech » defend une position similaire à la mienne. Gábor Betegh dans « What makes a myth eikos? » atténue la position de Burnyeat en insistant sur le fait que le Timée donne le meilleur récit de ce qu'a pu penser le fabricant de l'univers pendant qu'il le réalisait; d'une certaine façon on se débarrasse ainsi des Formes en en faisant des pensées de la divinité, comme dans le médio-platonisme.

Les autres articles portent sur la réception de Platon et d'abord chez Aristote. Dans «Should Aristotle have recognized final causes in Plato's Timaeus», Thomas K. Johansen estime qu'Aristote a raison de soutenir que, dans le Timée, Platon ne tient compte que de la cause formelle et de la cause matérielle, en négligeant la cause finale. Le démiurge fabrique le monde à partir d'éléments formels relevant de la géométrie. Or, dans le dernier livre de la Métaphysique, Aristote soutient que les mathématiques ne peuvent fournir aucune cause, ni nécessaire ni suffisante en vue d'assurer la bonté dans les choses. Le démiurge ne peut donc se soucier de la cause finale. En revanche, dans « Aristotle on Plato on Weight $\gg$, Alan Code veut montrer que, dans le De caelo IV 2, Aristote attribue à Platon une définition inexacte de la pesanteur, car elle se fonde sur Timée 56a sq.

PLATO, The electronic Journal of the International Plato Society, n 11, 2011.

http://gramata.univ-paris1.fr/Plato/article108.html

(C) All rights of reproduction of any form reserved. 
qui explique la pesanteur en terme de nombre plutôt que sur Timée 62c3-63e8 en terme de balance.

Dans «Cosmic cratfsmanship in Plato and Stoicism», Anthony Long, explique comment les Stoïciens pour rendre compte de la présence d'une raison providentielle dans le monde ont remplacé par l'action d'un logos impersonnel la figure du démiurge, une divinité personnalisée se situant hors du monde qu'il fabrique et qui permet à l'âme du monde de prendre le relais de son action; l'exposé est clair et précis, mais il reprend là une idée qui avait été brillamment développée il y a longtemps par Joseph Moreau (L'âme du monde de Platon aux Stoïciens, Paris, Les Belles Lettres, 1939). Dans « Maker or Father? The demiurge from Plutarch to Plotinus », Matthias Vorwerk fait l'inventaire des interprétations de cette formule que l'on trouve dans le Timée pour désigner le démiurge: «Cela dit, trouver le fabricant et le père de l'univers exige un effort et, lorsqu'on l'a trouvé, il n'est pas possible d'en parler à tout le monde » (28c), proposées par différents penseurs qualifiées de médio-platoniciens: Plutarque, Apulée et Numénius notamment pour finir par Plotin. Le démiurge est un père car il engendre le monde qui est un vivant, et c'est un fabricant parce que son travail est décrit à l'aide de termes techniques; l'intérêt de cet article se situe dans son ouverture à une littérature secondaire de langue étrangère. Dans «What's the matter? Some neoplatonist answers », Ian Mueller propose un inventaire des réponses à cette question, données par Proclus, Périclès de Lydie, Simplicius et Philopon.

Un certain nombre d'articles s'intéressent d'un point de vue littéraire et dramatique du couple que forment le Timée et le Critias. Dans un article intitulé: «A time for learning and for counting: Egyptians, Greeks, and empirical processes in Plato's Timaeus », Barbara M. Sattler veut comprendre le sens de la critique que fait le prêtre égyptien à Solon : «Solon, Solon, vous autres Grecs êtes toujours des enfants; vieux, un Grec ne peut l'être » (Timée 22b). Les Grecs ne savent appréhender le passé qu'en comptant, sans y chercher une leçon. Le Timée permet de répondre à cette critique en structurant, par l'intermédiaire de l'âme, la vie humaine sur le modèle du mouvement des astres. Dans « Narrative order in the Timaeus and the Critias », Kathryn A. Morgan, veut montrer comment le récit dans le Timée et dans le Critias sert à mettre en relation un modèle du monde et de la cité avec l'univers actuel et ce que devrait être la bonne cité réelle. Dans «Timaeus in tinseltown: Atlantis in film», Jon Solomon suit à la trace la

PLATO, The electronic Journal of the International Plato Society, n 11, 2011.

http://gramata.univ-paris1.fr/Plato/article108.html

(C) All rights of reproduction of any form reserved. 
réception du mythe depuis la renaissance jusqu'au cinéma le plus actuel (tinseltown renvoie à Hollywood) en passant par Jules Verne.

Le recueil s'achève sur deux visions plus contemporaines. Anthony Vidler, dans « The Atlantis effect. The lost origins of architecture », retrace l'influence qu'a pu avoir dans l'histoire de l'architecture la description faite par Platon des constructions de l'Atlantide. Ann Bergren, dans «Plato's Timaeus and the aesthetics of animate forms », essaie de déterminer les rapports que l'on peut établir entre le Timée et une tendance architecturale qualifiée de « animate form » et représenté par Greg Lynn et Elena Manferdini; pour une tentative similaire, voir L'architecture de la vie: de Platon à la tenségrité. The architecture of life: from Plato to tensegrity, Sciences et techniques en perspective série II, vol. 9, fasc. 2, 2005 (Brepols publishers). Enfin, Sean Carroll, dans « Time and change in an eternal universe » tente d'établir un lien entre le Timée et le Big Bang, comme j'ai tenté de le faire avec F. Walter Meyerstein dans Inventer l'univers. Le problème de la connaissance et les modèles cosmologiques, Paris, Les Belles Lettres, 1991 (traduction en anglais sous le titre Inventing the Universe, Albany, SUNY press,1995).

Tous les articles figurant dans ce recueil sont bien écrits et en général bien informés. Mais presque tous - leurs auteurs enseignent d'ailleurs dans des Universités anglaises ou américaines ou y sont de passage - se situent dans un contexte interprétatif qui est celui du monde anglo-saxon de l'après-guerre. La chose est évidente non seulement dans les thèmes traités, mais aussi dans la littérature secondaire. Peu d'articles citent des travaux en langue étrangère ou appartenant à une autre tradition; et notre plus grand regret est que nous ne trouvons aucune discussion prenant vraiment en compte des positions sinon opposées du moins différentes de celles exposées, probablement par manque d'espace.

Luc Brisson

CNRS, Paris (Villejuif)

PLATO, The electronic Journal of the International Plato Society, n 11, 2011.

http://gramata.univ-paris1.fr/Plato/article108.html

(C) All rights of reproduction of any form reserved. 